\title{
Atendimento domiciliar ao idoso: problema ou solução?
}

\section{Home care for the elderly: problem or solution?}

\author{
1 Escola Nacional de Saúde \\ Pública, Fundação Oswaldo \\ Cruz, Rio de Janeiro, Brasil. \\ Correspondência \\ C. A. Floriani \\ Escola Nacional \\ de Saúde Pública, \\ Fundação Oswaldo Cruz. \\ Rua Leopoldo Bulhões 1480 \\ Rio de Janeiro, $R J$ \\ 21041-210, Brasil. \\ cirofloriani@terra.com.br
}

\begin{abstract}
Home care for the elderly has become an important health care tool in both developed and developing countries. However, several ethical, social, and operational concerns have received insufficient attention, and the Brazilian literature on this theme is limited. Starting with a bibliographic review on home care, this paper takes a bioethical approach to potential problems arising from this growing and important patient care modality. A broader ethical approach is needed to implement home care for the elderly, with policies to protect the patient, family, and caregiver, aimed at improving the quality of this program format.
\end{abstract}

Bioethics; Aged; Family; Medical Care; Aging Health
Ciro Augusto Floriani 1

Fermin Roland Schramm 1

\section{Introdução}

O crescimento do atendimento domiciliar (AD) no Brasil é recente, datando da última década do século XX ${ }^{1}$. A difusão desta modalidade de prestação de serviços ocorre tanto no setor privado quanto no setor público, fazendo parte da pauta de discussão das políticas de saúde que, pressionadas pelos altos custos das internações hospitalares, buscam saídas para uma melhor utilização dos recursos financeiros 2 .

$\mathrm{O} \mathrm{AD}$ envolve vários atores sociais em uma rede complexa de relações, gerando situações de conflitos de interesses e de valores entre esses atores 3,4 . Neste artigo, iremos, inicialmente, conceituar o AD e procuraremos contextualizá-lo em nossa realidade para, em seguida, descrevermos aspectos problemáticos e conflituosos, do ponto de vista moral, a partir de revisão da literatura que aborda questões éticas em relação ao $\mathrm{AD}$, a maior parte dela com enfoque centrado no idoso. Discutiremos alguns desses conflitos dentro de uma perspectiva da bioética de proteção, concluindo pela necessidade de elaborar uma agenda que considere os atores mais vulneráveis neste processo, ou seja, o paciente, sua família e o cuidador. Com isso, esperamos estar contribuindo para o aperfeiçoamento do $\mathrm{AD}$, no momento em que este passa a ser incluído na Política Nacional do Idoso com a criação da Assistência Domiciliar Geriátrica (http://www.saude.gov.br/aude/apli 
cac.../noticias_detalhes.ctm?co_seq_noticias= 290 , acessado em 25/Nov/2002) e a ser disciplinado pelo Conselho Federal de Medicina (http: //www.portalmedico.org.br/resolucoes/cfm/ 2003/1668_2003.htm, acessado em 27/Jun/2003).

\section{Definindo atendimento domiciliar}

O termo AD é aqui empregado no sentido amplo de home care, compreendendo uma gama de serviços realizados no domicílio e destinados ao suporte terapêutico do paciente. Estes serviços vão desde cuidados pessoais de suas atividades de vida diária (higiene íntima, alimentação, banho, locomoção e vestuário), cuidados com sua medicação e realização de curativos de ferimentos, cuidados com escaras e ostomias, até o uso de alta tecnologia hospitalar como nutrição enteral/parenteral, diálise, transfusão de hemoderivados, quimioterapia e antibioticoterapia, com serviço médico e de enfermagem 24 horas/dia, e uma rede de apoio para diagnóstico e para outras medidas terapêuticas. Também estão incluídos neste conceito o chamado suporte comunitário (voluntários, serviços de associações comunitárias, transporte) e realização de tarefas externas, como ida a um banco ou a uma farmácia 4 .

\section{Objetivos do atendimento domiciliar}

Os objetivos do AD são: contribuir para a otimização dos leitos hospitalares e do atendimento ambulatorial, visando a redução de custos; reintegrar o paciente em seu núcleo familiar e de apoio; proporcionar assistência humanizada e integral, por meio de uma maior aproximação da equipe de saúde com a família; estimular uma maior participação do paciente e de sua família no tratamento proposto; promover educação em saúde; ser um campo de ensino e pesquisa 5 . Referindo-se ao idoso, o AD insere-se dentro de um modelo gerontológico que visa, na medida do possível, a reinserir o idoso na comunidade, preservando ao máximo sua autonomia, buscando a recuperação de sua independência funcional 2 e procurando mantê-lo um “cidadão - ativo, participativo, produtivo e afetivo" 6 (p. 97). Assim, os potenciais benefícios com o $\mathrm{AD}$ seriam a diminuição das reinternações e dos custos hospitalares; a redução do risco de infecção hospitalar; a manutenção do paciente no núcleo familiar e o aumento da qualidade de vida deste e de seus familiares 7,8. Além do mais, o domicílio oferece- ria um lugar seguro ao idoso, protegendo-o do meio, evitando com isso sua institucionalização, tanto em nível hospitalar quanto asilar 4. Já os critérios de elegibilidade para a aceitação no AD costumam levar em consideração os seguintes aspectos: "estabilidade clínica; rede de suporte social (cuidador, família, amigos, voluntários etc.); ambiente apropriado; avaliação profissional das demandas existentes; suporte financeiro" 9 (p. 10).

\section{Contextualização do atendimento domiciliar}

Os pacientes, que até pouco tempo atrás eram tratados basicamente nos hospitais, são agora transferidos o mais rapidamente possível para seus domicílios, onde continuarão seus tratamentos, com programas de reabilitação, de recuperação ou de cuidados paliativos. Com o passar do tempo surgem situações conflituosas entre os diversos atores envolvidos: o paciente, a família, os cuidadores, o provedor e o gestor de saúde, envolvendo processos decisórios difíceis 4 . Estas situações de tensão e de conflito emergem dentro de um contexto maior onde alguns aspectos devem ser levados em consideração: (1) há uma crescente tendência dos administradores hospitalares em otimizar as internações e reinternações, pressionados pelos altos custos, com uma maior racionalização do tempo de permanência do paciente no hospital, maior rotatividade dos leitos, diminuição dos custos hospitalares e, nesse sentido, para o gestor, o AD apresenta-se como sendo uma opção importante 1,2; (2) há um modelo de formação médica centrado no hospital, com uma gama de recursos à disposição do médico, sendo este um local onde ele tende a se sentir mais familiarizado e mais seguro para atuar; (3) existe uma concepção amplamente difundida na comunidade de ser o hospital lugar de excelência para diagnóstico e tratamento; (4) o domicílio é um local onde o poder estabelecido pertence ao paciente ou aos membros daquela família, não sendo, portanto, território natural de atuação do profissional de saúde 10; (5) o sistema de institucionalização asilar é freqüentemente associado a abandono, confinamento e exclusão social, gerando reflexos negativos na sociedade, o que leva muitas famílias a optarem pela permanência do idoso no domicílio 11; (6) há uma população que vive em uma realidade geográfica e social muitas vezes desfavorável, em áreas de difícil acesso e com domicílios em precárias condições, o que 
pode trazer, como conseqüência, a exclusão de pacientes que poderiam se beneficiar com o $\mathrm{AD}$; (7) a mulher, que como veremos adiante costuma ser um dos atores fundamentais no $\mathrm{AD}$, representa, na atualidade, importante esteio financeiro familiar, o que pode comprometer sua atividade de cuidadora e vice-versa, a atividade de cuidadora podendo vir a interferir no seu emprego.

Dentro desta perspectiva, podemos perceber que o campo do $\mathrm{AD}$, onde interagem seus diferentes atores, carrega conflitos de natureza moral, e alguns desses conflitos pretendemos apresentar a seguir.

\section{A bioética da proteção}

\section{e $o$ atendimento domiciliar}

Considerando que a bioética surgiu em resposta aos crescentes conflitos e dilemas de natureza moral relacionados, dentre outras práticas, com ações no âmbito das políticas de saúde e com os avanços tecnológicos aplicados ao setor saúde 12,13,14 e que, para alguns autores, constitui um campo "pluridisciplinar" 15 ou "interdisciplinar" 16, no qual se utilizam várias ferramentas disciplinares para tentar compreender e resolver os conflitos morais que surgem, nas sociedades complexas e diferenciadas contemporâneas, das ações humanas sobre qualquer sistema e processo vivo, pode-se razoavelmente afirmar que a bioética visa a proteger os "pacientes morais" contra os efeitos daninhos e irreversíveis que podem resultar dos atos dos "agentes morais” 17. Por isso, tal concepção de bioética pode aplicar-se também às práticas do AD.

Com efeito, sabe-se que o AD é visto como uma alternativa à internação hospitalar prolongada e aos crescentes custos associados, conforme já salientado. Assim, a implantação do $\mathrm{AD}$, tanto na esfera pública quanto na privada, torna-se cada vez mais uma realidade em nosso meio, com uma importante dimensão moral, visto que pode implicar conseqüências daninhas e existencialmente irreversíveis para os doentes ou "pacientes morais". No caso específico em exame aqui, o processo decisório pode ser descrito como um cenário de escassez de recursos, no qual o gestor da saúde deve trabalhar e mostrar resultados, tomando decisões pautadas por indicadores econômicos e que devem, portanto, ser respeitadas por qualquer gestor que se queira tecnicamente "pragmático", isto é, preocupado com a otimização dos meios efetivamente disponíveis e que pretenda, também, atuar de forma moralmente "legítima", isto é, aceitável por qualquer agente social razoável e imparcial. No entanto, dentro desta perspectiva bioética, há questões que mereceriam ser aprofundadas devido a seu caráter problemático.

\section{Aspectos moralmente problemáticos \\ identificados ao longo da implantação \\ dos programas de atendimento domiciliar com respeito ao cuidador}

Já está bem estabelecido o perfil do cuidador informal de idosos: costuma ser do sexo feminino, filha ou esposa (muitas vezes idosa) que, com freqüência, divide esta atividade com seus afazeres diários como, por exemplo, cuidar das crianças; além disso, sabe-se que em algum momento esse cuidador pode ter problemas com seu emprego ou mesmo perdê-lo 18. Podemos imaginar o ônus desta árdua e desgastante tarefa, forjada numa repetitividade diária incessante, muitas vezes durante anos, com sobrecarga de atividades no seu cotidiano, sendo quase sempre uma atividade solitária e sem descanso, que pode levá-lo a um isolamento afetivo e social 19. Soma-se a isto o fato de, em determinadas situações, o cuidador sentir-se enganado e abandonado pelo provedor do $\mathrm{AD}$, principalmente quando esses cuidados ocorrem por vários anos seguidos 20 . Sabe-se, também, que o cuidador enfrenta rupturas de vínculos, tem sua saúde deteriorada, não tem férias e tem baixa participação social 21 . É descrita, também, a perda do poder aquisitivo da família, com a progressão da doença do paciente 22. Muitas cuidadoras, principalmente aquelas que trabalham fora de casa, dividem a função de cuidadora com crianças e adolescentes, que passam a ser os responsáveis pela maior parte dos cuidados diários 18,23. Para o cuidador, portanto, trata-se não só de uma sobrecarga nas atividades, mas também de uma ameaça à sua saúde, já que muitos adoecem ou agravam problemas de saúde já existentes. Porém, não só os aspectos negativos são relevantes, ainda que sejam os mais estudados, mas transformações positivas são relatadas por cuidadores que passaram a ter suas vidas modificadas, dando a elas um sentido até então inexistente, com uma experiência interior de crescimento e de transformação 3,24 .

A relação entre o cuidador e o idoso dependente é complexa e, dependendo do perfil psicológico de ambos, poderá ser muito difícil, principalmente em relação à autonomia do idoso que, apesar de estar dependente e frágil, muitas vezes tem expectativas de exercitá-la tão plenamente quanto em seu passado 25 . O cuidador pode interpretar erroneamente, na continuidade repetitiva dos cuidados diários, 
que a inabilidade de um idoso em fazer algo seja igual à incapacidade dele tomar decisões. Com isso, pode impor seu modo de realizar as atividades, não ouvindo os desejos e anseios legítimos do idoso, com erosão da autonomia deste e acentuação de um modelo paternalista de cuidados: "os domicílios são os lugares naturais e escolas para práticas paternalistas” 26 ( $\mathrm{p}$. 170). É claro que o "paternalismo" nem sempre é um problema já que, muitas vezes, refere-se a práticas que em princípio visam a proteger o paciente; porém, no caso do cuidador não considerar a vontade do idoso competente e não respeitar o exercício de sua autonomia, esta situação deve ser encarada como sendo moralmente problemática. Com efeito, este modo de agir tende a ser a tônica da relação principalmente levando-se em conta o longo tempo em que duram tais cuidados, podendo o paternalismo ser acentuado pela tendência de se olhar para o idoso como infantilizado em suas atitudes e desejos 4,26. Neste sentido, abusos de poder por parte do cuidador têm sido relatados, tais como agressões, impaciência e ressentimentos, e o fato de o cuidador ser contratado também não assegura, necessariamente, um atendimento livre dessas idiossincrasias.

Uma situação especialmente preocupante diz respeito ao idoso cuidar de idoso, situação descrita como sendo a de "idoso jovem cuidando de idoso idoso" 18 (p. 625). Podendo estar também acometido por múltiplas co-morbidades, esse cuidador idoso irá encontrar dificuldades para conseguir cuidar do paciente, descuidando de sua própria saúde e, assim, comprometendo e agravando a condição clínica de ambos.

\section{- Questões morais referentes ao cuidador}

Pode-se, por exemplo, perguntar o quanto as atividades diárias do cuidador, forjadas na repetitividade, muitas vezes de anos, são de fato benéficas para o paciente idoso, visto que elas pretenderiam sempre, em última instância "proteger" o real, ou suposto, beneficiário do ato de cuidado? Como ações paternalistas, freqüentemente presentes nas ações dos cuidadores, podem ser transformadas em ações efetivamente terapêuticas e até onde elas implicam, de fato, seqüelas no idoso? Por outro lado, quem arcará com os potenciais danos físicos, emocionais, sociais e existenciais do cuidador? Em outras palavras, será que, nesses casos, o paternalismo pode ser visto como sendo também protetor, de fato, de todos os envolvidos pelo ato do cuidar, sendo, por isso mesmo, moralmente justificado? Ou será que paternalismo e proteção são de fato antinômicos, visto que a práti- ca correta dos pais com os filhos implicaria também o tornar a criança progressivamente autônoma, capaz de tomar suas decisões, ao passo que o paternalismo seria de fato a negação desta competência?

\section{Aspectos moralmente problemáticos identificados ao longo da implantação dos programas de atendimento domiciliar com respeito ao provedor do atendimento domiciliar}

Para Collopy et al. ${ }^{4}$, os pacientes estariam sendo deslocados de modo mais rápido e mais doentes para o domicílio - quicker and sicker devido aos custos das internações hospitalares. Arras \& Dubler 3 descrevem situações de tensão e conflitos vivenciadas pelo paciente e sua família em relação ao tempo de permanência hospitalar, bem como dificuldade destes em compreenderem o significado e o valor do AD, oferecido como alternativa viável e segura.

A propaganda utilizada pelo provedor do AD junto ao usuário incorpora a mesma tecnologia biomédica utilizada pela propaganda hospitalar, numa tentativa de demonstrar ser o domicílio tão seguro, ou mais, do que o hospital 3. Além disso, profissionais de saúde formados com enfoque intervencionista podem trazer problemas no domicílio, agindo por ansiedade e por sentirem a necessidade de terem de fazer alguma coisa, podendo gerar um ambiente de desconfiança e de tensão 4 .

Há famílias e pacientes que impõem condições ao provedor do $\mathrm{AD}$ com respeito ao perfil racial e de classe do cuidador que passará a conviver em seus domicílios 4,21, com potenciais conflitos de natureza discriminatória.

\section{- Questões morais referentes ao provedor do atendimento domiciliar}

De que modo, e até onde, têm sido levadas em consideração as escolhas "autônomas" do paciente idoso, partindo-se da premissa ética de que a preservação da autonomia do paciente, durante o maior tempo possível, pode nortear moralmente o $\mathrm{AD}$ gerontológico, visto que, em última instância, o que conta é o potencial benefício do paciente, objeto real, ou suposto, do cuidado? Que medidas de suporte e amparo são de fato oferecidas pelos programas de $\mathrm{AD}$ e que medidas protetoras estarão nas pautas de deliberação dos gestores da saúde com relação ao paciente, à família e ao cuidador? Será legítimo, do ponto de vista moral, que sejam executadas estratégias de convencimento junto ao usuário, movidas por objetivos que podem es- 
tar vinculados, única e exclusivamente, à redução de custos, conduzindo ao surgimento de um mercado de reserva lucrativo como único parâmetro, logo, a uma espécie de reducionismo econômico da complexidade do real sanitário, que pode ser vista, por exemplo, como uma injustificável "tirania" de uma esfera de justiça sobre as demais 27 ? Uma vez implantado o AD do tipo internação domiciliar, como ficará o acesso a ele pela população mais desfavorecida, muitas vezes com domicílios sem as mínimas condições para sua instalação? A quem, de fato, se destina esse tipo específico de $\mathrm{AD}$ e a quem ele deveria, legitimamente, se destinar? Ainda dentro do contexto da internação domiciliar, qual é a qualificação do profissional de enfermagem que fica no domicílio e, que tipo de vínculo esse profissional e o médico estabelecem com o paciente e a família? Como serão possíveis diagnósticos precoces e, por conseguinte, intervenções bem sucedidas se, muitas vezes, estão no domicílio profissionais de enfermagem despreparados para fazer diagnósticos e se não há visitas médicas diárias a esses pacientes, como seria de se esperar quando há uma internação?

\section{Aspectos moralmente problemáticos identificados ao longo da implantação dos programas de atendimento domiciliar com respeito ao domicílio}

Segundo Arras \& Dubler 3, o fato realmente novo com o advento do $\mathrm{AD}$, principalmente em relação ao uso de alta tecnologia, consiste na “hipermedicalização" deste domicílio. Para esses autores, essa transferência das ações médicas para a vida privada levanta a questão de "que tipo de casa e de família nós queremos que a sociedade adote, e a que preço?", pois corremos o risco de tornar alguns domicílios em " $m e$ ros satélites das instituições médicas” 3 (p. S20). Com isso, o AD corre o risco de ser encarado apenas como uma mudança de local de tratamento e não como uma mudança de filosofia nos cuidados administrados ao idoso 4 . Com efeito, a transformação do domicílio pela internação domiciliar pode levar o AD a ser "um complexo fenômeno social que melhora a vida para muitos pacientes graves, minando para outros as condições que tendem a promover importantes benefícios sociais e oportunidades" 3 (p. S20).

\section{- Questões morais referentes ao domicílio}

De que modo e quanto a experiência de um domicílio adaptado ao tratamento com alta tecnologia hospitalar irá afetar as pessoas que vivem nesse domicílio? Não se estará invadindo e desorganizando este espaço íntimo, onde as relações cotidianas daquela família foram construídas? Ainda dentro desse contexto: é lícito que se transfira alta tecnologia médica aos domicílios, com tomadas de decisão direcionadas por modelos intervencionistas, tornando, com isso, os domicílios meros "satélites" dos hospitais, mas sem a necessária competência para atuar, de fato, como tais? Ou: que tipos de acordos podem ser e são moralmente legítimos entre a equipe do $\mathrm{AD}$ e a família, já que esta detém o poder por estar em seu território?

\section{Outros aspectos morais geradores de conflitos}

O paciente e a família estariam sentindo-se pressionados pelos planos de saúde para adesão ao $\mathrm{AD}{ }^{3}$. Segundo Ruddick 26 , há pacientes que preferem ficar e serem cuidados num ambiente hospitalar e dever-se-ia, portanto, respeitar este desejo, principalmente quando a doença está em curso avançado, com deterioração física.

Em relação à adesão médica, pelo menos nos países desenvolvidos, há uma certa resistência ao AD. Os fatores apontados para isto são a baixa remuneração, a inconveniência quanto ao deslocamento e ao tempo demandado, a qualidade dos cuidados no domicílio, os aspectos legais relacionados a determinados procedimentos médicos no domicílio e a perda do poder e de controle 3,28 . Além disso, estaria havendo uma fragmentação das responsabilidades quanto aos procedimentos no domicílio, com enfraquecimento da relação médico/paciente, justamente o oposto do apregoado pela propaganda sobre os benefícios do $\mathrm{AD}$, a qual sugere que o $\mathrm{AD}$ fortalece esse vínculo. No contexto dos cuidados a longo prazo no domicílio, os enfermeiros estariam sentindo-se menos pressionados do que nas instituições hospitalares, obtendo uma maior carga de responsabilidade e de autoridade 3 , desenvolvendo um "olhar próprio" 10.

Os programas de AD de longa permanência não necessariamente reduzem os custos, mas podem trazer benefícios para o paciente, a família e para o cuidador, tais como aumento na satisfação com a qualidade de vida e aumento na atenção e na confiança em relação aos cuidados domiciliares. Esta foi uma das conclusões do National Long Term Care Demonstration 29, estudo multicêntrico realizado nos Estados Unidos em 1982, que avaliou, durante um ano e seis meses, 6 mil idosos fragilizados inseridos em um $\mathrm{AD}$ do tipo case management (atendimento médico ambulatorial, suporte 
telefônico contínuo e visitas no domicílio). Para Weissert 30, esses programas deveriam ser baseados nos seus benefícios ao usuário e não na possibilidade de redução de custos. No Brasil ainda não temos, até a presente data, estudos desta magnitude.

Diante do exposto e voltando aos objetivos do AD descritos por Osmo \& Castellanos 5, podemos constatar que: a otimização dos leitos e a redução de custos hospitalares, em nosso meio, é uma necessidade premente e que o $\mathrm{AD}$ do tipo internação domiciliar parece estar se mostrando como sendo uma alternativa viável, sob este prisma; a reorganização da rede ambulatorial faz-se necessária e o $\mathrm{AD}$ do tipo case management poderia ser uma alternativa já que, comprovadamente, traz significativos benefícios ao usuário, ainda que com acréscimo nos custos; a reintegração do paciente em seu núcleo familiar e de apoio nem sempre é desejada pelo usuário e, no caso do idoso, pode sofrer resistência por parte da família impondo a esta um ônus significativo; a delicada relação entre o cuidador e o idoso dependente mostra que a humanização (palavra invariavelmente encontrada nos textos, sem ser exatamente definida) está, muitas vezes, longe de acontecer e que o domicílio e a proximidade da equipe de saúde não são garantias disto. Acreditamos que não é o domicílio e, portanto, não é necessariamente o local que humaniza a relação terapêutica, mas, sim, o interesse empático que o profissional desenvolve pelo paciente. Assim sendo, surge a pergunta: a que preço, com que propósito e a quem cabe o ônus de oferecer à família uma maior participação no tratamento? Durante os meses ou anos seguintes continuará a equipe do $\mathrm{AD}$ a dar suporte ou tenderá a acomodar-se, gerando sensação de exclusão e de abandono nesses familiares?

\section{- Questões morais relacionadas à dignidade do morrer}

Em casos já diagnosticados como irreversíveis, de acordo com a melhor "ciência" médica existente, não se estarão transferindo e perpetuando no domicílio ações intervencionistas que prolongam a vida indefinidamente, sem necessariamente conservar, também, sua qualidade, com decisões que poderiam ter sido discutidas, de maneira mais apropriada, no hospital? $\mathrm{Ou}$, em casos de progressão da doença de base, com irreversibilidade do quadro clínico, levando à perda importante da qualidade de vida do paciente e a despeito da otimização medicamentosa, qual deverá ser a conduta, ao mesmo tempo pragmaticamente eficaz e moralmente legítima, que seja norteadora da equipe do $\mathrm{AD}$, em especial a do médico assistente? Questões como estas, que surgem no contexto do $\mathrm{AD}$, dizem respeito ao suporte da vida, como, por exemplo, a manutenção de nutrição enteral/parenteral 31 ou ao quanto deve ser razoavelmente investido no diagnóstico e tratamento da doença ou de complicações que possam surgir 32,33; dizem respeito, também, à dignidade do morrer, e em relação a este aspecto, acreditamos que a questão principal não deva ser onde se morre, mas, sim, como e de que modo se morre. Ou ainda, a um olhar médico crítico e criterioso em relação à "polifarmácia”, freqüentemente utilizada, com a conseqüente iatrogenia medicamentosa, fato muito comum na população idosa, agravando ainda mais o já delicado estado clínico do paciente 34 . O que se sugere aqui é uma reflexão sobre se é possível salvar essa vida, com que qualidade, e, na total impossibilidade disto, nos dizeres de uma médica intensivista, o quanto é possível "o que eu tenho chamado de salvar uma morte: a ajudar o paciente a preservar conforto e dignidade, apesar da doença esmagadora, a ajudar a família a compreender a inevitabilidade da morte e como a seu tempo ela pode ser apropriada, e como se encaminhar no processo de luto. Salvar mortes, eu dei-me conta, é tão importante e gratificante quanto salvar vidas" 35 (p. 777).

Sendo o AD um modelo de prestação de serviços estruturado no contexto das políticas de saúde, destinado a uma população com especificidades bem definidas como, por exemplo, os doentes crônicos idosos, e devido ao fato de encerrar em seu desenvolvimento uma série de implicações éticas e sociais ${ }^{3}$, fruto das inter-relações entre seus diversos atores, podese acreditar que este campo constitui, de modo enfático, um âmbito privilegiado de políticas de proteção, que devem ser pensadas e realizadas, contemplando os grupos de maior "vulnerabilidade", isto é, os de fato mais desamparados. No caso específico do AD, entendemos que este caráter protetor deva ser voltado para o paciente e sua família, onde freqüentemente é "gestado" o cuidador. Isto parece ser especialmente relevante quando se está diante de pacientes idosos com doenças terminais, muitas vezes envoltos em um ambiente de frieza, sofrimento e abandono.

\section{Conclusão}

Como tentamos mostrar, os cuidados administrados no domicílio suscitam importantes questões bioéticas, visto que dizem respeito a 
ações que podem ter efeitos irreversíveis indesejáveis sobre os destinatários dos atos do AD. Por isso, pensamos que, pelo menos em parte, elas poderiam ser minimizadas se os médicos tivessem por hábito conversar abertamente com os pacientes sobre suas expectativas de vida, sobre a morte e sobre um uso razoável de tecnologia no domicílio, ajudandoos na melhor escolha possível para sua qualidade de vida 36 .

Para tanto, diante do crescimento significativo do AD na última década, é preciso distinguir programas que simplesmente aumentam a carga de cuidados nos domicílios, daqueles que se preocupam com a qualidade de tais cuidados e com as conseqüências que podem resultar deste tipo de abordagem 37 . Por isso, torna-se necessária a construção de uma melhor agenda de políticas de fiscalização do $\mathrm{AD}$, que esteja voltada para nossa realidade, com dados mais consistentes sobre sua segurança, eficácia e efetividade, além de maiores estudos relacionados à qualidade de vida de seus usuários e aos aspectos bioéticos envolvidos. Em outros termos, não basta apenas preparar um cuidador do ponto de vista técnico, condição certamente necessária do ponto de vista da aceitabilidade de uma prática que possa ser considerada "competente", mas é preciso, também, estruturar intervenções junto a esse cuidador, que possam ser consideradas, também, "legítimas", de acordo com os anseios morais existentes e resultantes do sentimento de desamparo que afeta concretamente quem sofre e não quer, por boas razões, "entregar os pontos". Com efeito, de acordo com Topinková 21 , tais intervenções devem ser pensadas, programadas e estabelecidas em diferentes níveis (físico, psicológico, social e financeiro) e não basta apenas ter uma equipe para atuar, mas é ainda necessário saber que tipo de intervenção é pretendido e se essa intervenção é, além de pragmaticamente desejável, também moralmente legítima.

Assim sendo, é preciso ter um olhar crítico sobre o AD, ponderando seus aspectos positivos e negativos do ponto de vista da efetiva proteção dos usuários, tendo, em particular, consciência de que, na pauta das definições e nos processos decisórios de sua implantação, os princípios éticos e bioéticos sejam de fato considerados 3,30,38. Neste âmbito, acreditamos que políticas que sejam efetivas pragmaticamente e legítimas moralmente, de acordo com o ethos social vigente, isto é, que protejam de fato os agentes mais desamparados, são imprescindíveis para abarcarem as múltiplas questões geradas pelo $\mathrm{AD}$. Consideramos também que, do ponto de vista da corroboração, ou da refutação, da pertinência de uma bioética da proteção aqui proposta, seja necessário um maior número de trabalhos qualificados, que busquem aprofundar a discussão sobre os possíveis desdobramentos práticos do $\mathrm{AD}$, contribuindo, com isto, para o aperfeiçoamento desse modelo terapêutico.

Em relação ao $\mathrm{AD}$ oferecido como parte das estratégias dos cuidados no fim da vida (com uma referência especial aos doentes com câncer, mas, evidentemente, não se restringindo a eles) sabemos que este é um campo fértil para ações compassivas e acreditamos que a bioética da proteção tem aqui importante contribuição, ajudando na construção de programas de $\mathrm{AD}$ que de fato protejam esses pacientes, norteando esses programas para a construção de modelos onde as condutas dos profissionais envolvidos, em especial a dos médicos, sejam não somente corretas do ponto de vista técnico (imprescindíveis), mas também amorosas e empáticas.

Para concluir e para "visualizar" melhor o que deveria ser feito, acreditamos que é preciso nos perguntarmos com que objetivo estamos entrando no domicílio de alguém que precisa de nossa ajuda, sendo que devemos voltar nosso olhar para o entendimento das reais necessidades do idoso e daquela família envolvidos, numa época em que as diretrizes econômicas parecem ocupar papel preponderante nas tomadas de decisão, muitas vezes desconsiderando a própria vocação histórica da economia, a qual, como diz a filologia, significa "a norma (moral) que (deve) guiar o ambiente (onde vivemos)". Por isso, aquilo que irá vigorar vai depender, fundamentalmente, das intenções e atitudes dos "agentes morais" que somos ou que deveríamos ser para "pôr ordem" em nossas inter-relações entre "agentes" e "pacientes" morais, isto é, entre quem tem por vocação proteger e quem deve ser protegido. 


\section{Resumo}

O atendimento domiciliar ao idoso tem se tornado um importante instrumento de assistência nos últimos anos, tanto nos países desenvolvidos quanto nos países em desenvolvimento. Vários aspectos éticos, sociais e operacionais têm sido negligenciados e a literatura nacional é escassa em relação a esta temática. A partir de revisão bibliográfica em atendimento domiciliar, este artigo enfoca, do ponto de vista bioético, os potenciais problemas advindos com a implantação dessa crescente e importante modalidade de atendimento. Conclui ser necessário um maior direcionamento ético na implantação do atendimento domiciliar, com políticas de proteção ao paciente, à família e ao cuidador, visando a aperfeiçoar a qualidade dos programas oferecidos.

Bioética; Idoso; Família; Cuidados Médicos; Saúde do Idoso

\section{Colaboradores}

C. A. Floriani contribuiu na elaboração do artigo. F. R. Schramm colaborou na discussão e revisão final do artigo.

\section{Referências}

1. Mendes W. Home care: uma modalidade de assistência à saúde. Rio de Janeiro: Universidade Aberta da Terceira Idade, Universidade do Estado do Rio de Janeiro; 2001.

2. Gordilho A, Sérgio J, Silvestre J, Ramos LR, Freire MPA, Espindola N, et al. Desafios a serem enfrentados no terceiro milênio pelo setor saúde na atenção integral ao idoso. Rio de Janeiro: Universidade Aberta da Terceira Idade, Universidade do Estado do Rio de Janeiro; 2000.

3. Arras J, Dubler NN. Bringing the hospital home: ethical and social implications of high-tech home care. Hastings Cent Rep 1994; 24:S19-28.

4. Collopy B, Dubler N, Zuckerman C. The ethics of home care: autonomy and accommodation. Hastings Cent Rep 1990; 20 Suppl:S1-16.

5. Osmo AA, Castellanos PL. Os cuidados a domicílio: da decisão política à gestão de programas. http:/www.ufrgs.br/pdgs/Cuidadomicilio.htm (acessado em 03/Abr/2000).

6. Sayeg MA. Envelhecimento bem sucedido e o autocuidado: algumas reflexões. Arquivos de Geriatria e Gerontologia 1998; 2:96-8.

7. Dolben LW. Serviço de atendimento de enfermagem residencial. In: Duarte YAO, Diogo MJ, organizadores. Atendimento domiciliário - um enfoque gerontológico. São Paulo: Atheneu; 2000. p. 575-82.

8. Monteiro CP, Monteiro JL. Internação domiciliária. In: Duarte Y, Diogo M, organizadores. Atendimento domiciliário - um enfoque gerontológico. São Paulo: Atheneu; 2000. p. 584.

9. Duarte YAO, Diogo MJ. Atendimento domiciliário um enfoque gerontológico. São Paulo: Atheneu; 2000.

10. Liaschenko J. The moral geography of home care. ANS Adv Nurs Sci 1994; 17:16-26.

11. Pavarini SC, Neri AL. Compreendendo dependência, independência e autonomia no contexto domiciliar: conceitos, atitudes e comportamentos. In: Duarte Y, Diogo M, organizadores. Atendimento domiciliário - um enfoque gerontológico. São Paulo: Atheneu; 2000. p. 49-69.

12. Jonsen AR. A chronicle of ethicl events: 1940s to 1980s. In: Jonsen AR, editor. A short history of medical ethics. New York/Oxford: Oxford University Press; 2000. p. 99-114.

13. Kuhse H, Singer P. What is bioethics? A historical introduction. In: Kuhse H, Singer P, editors. A companion to bioethics. Oxford: Blackwell Publishers; 1998. p. 3-11.

14. Mori M. A bioética: sua natureza e história. Humanidades 1994; 9:333-41.

15. Hottois G. Bioéthique. In: Hottois G, Missa JN, editor. Nouvelle encyclopédie de bioéthique. Bruxelas: De Boeck; 2001. p. 124-31.

16. Ladrière J. Del sentido de la bioética. Acta Bioethica 2000; 6:199-218.

17. Schramm FR. Bioética para quê? Revista Camiliana da Saúde 2002; 1:14-21.

18. Stone R, Cafferata GL, Sangl J. Caregivers of the frail elderly: a national profile. Gerontologist 1987; 27:616-26.

19. Brody EM. "Women in the middle" and family help to older people. Gerontologist 1981; 21:471-80. 
20. Levine C. The loneliness of the long-term care giver. N Engl J Med 1999; 340:1587-90.

21. Topinková E. Family caregiving for the elderly: are there ways to meet the need? In: Callahan D, ter Meulen RHJ, Tupinková E, editors. A world growing old: the coming health care challenges. Washington DC: Georgetown University Press; 1995. p. 106-16.

22. George LK, Gwyther LP. Caregiver well-being: a multidimensional examination of family caregivers of demented adults. Gerontologist 1986; 26:253-9.

23. Brody E. Parent care as a normative family stress. Gerontologist 1985; 25:19-29.

24. Mendes PMT. Cuidadores: heróis anônimos do cotidiano. In: Karsch UM, organizador. Envelhecimento com dependência: revelando cuidadores. São Paulo: Educ; 1998. p. 171-97.

25. Neri AL. Bem-estar e estresse em familiares que cuidam de idosos fragilizados e de alta dependência. In: Neri AL, organizador. Qualidade de vida e idade madura. Campinas: Papirus Editora; 2000. p. 237-85.

26. Ruddick W. Transforming homes and hospitals. In: Arras J, editor. Bringing the hospital home: ethical and social implications of high-tech home care. Baltimore: The Johns Hopkins University Press; 1995. p. 166-79.

27. Walzer M. Spheres of justice: a defense of pluralism and equality. New York: Basic Books; 1983.

28. Keenan JM, Fanale JE. Home care: past and present, problems and potencial. J Am Geriatr Soc 1989; 37:1076-83.

29. Kemper P. The evaluation of the national long term care demonstration 10. Overview of the findings. Health Serv Res 1988; 23:161-74.
30. Weissert WG. The national channeling demonstration: what we knew, know now and still need to know. Health Serv Res 1988; 23:175-87.

31. Vernon MJ. Decisions on life-sustaining therapy: nutrition and fluid. In: Rai GS, editor. Medical ethics and the elderly: practical guide. Amsterdam: Harwood Academic Publishers; 1999. p. 3953.

32. American Medical Association. Medical futility in end-of-life care: report of the council on ethical and judicial affairs. JAMA 1999; 281:937-41.

33. Emanuel EJ, Emanuel LL. The promise of a good death. Lancet 1998; 351 Suppl 2:21-9.

34. Nascimento JSF. Farmacologia e terapêutica na velhice. In: Freitas EV, Py L, Neri AL, Cançado FAX, Gorzoni ML, Rocha SM, organizadores. Tratado de geriatria e gerontologia. Rio de Janeiro: Guanabara Koogan; 2002. p. 618-23.

35. Nelson JE. Saving lives and saving deaths. Ann Intern Med 1999; 130:776-7.

36. Pousada L. High-tech home care for elderly persons: what, why and how much? In: Arras J, editor. Bringing the hospital home: ethical and social implications of high-tech home care. Baltimore: The Johns Hopkins University Press; 1995. p. 107-28.

37. Barusch AS. Programming for family care of elderly dependents: mandates, incentives, and service rationing. Soc Work 1995; 40:315-22.

38. Kanoti GA. Home care: a shifting of ethical responsabilities. Cleve Clin Q 1985; 52:351-4.

Recebido em 30/Jun/2003

Versão final reapresentada em 09/Dez/2003 Aprovado em 08/Mar/2004 